Lígia Leandro N unes Serpa'

Katjy Veiga Rosário Martins Costa'

Júlio Cesar Voltolini"

Iná Kakitani"'I

\section{Variação sazonal de Aedes aegypti e Aedes albopictus no município de Potim, São Paulo}

\author{
Seasonal variation of Aedes aegypti \\ and Aedes albopictus in a city of \\ Southeastern Brazil
}

\section{RESU MO}

OBJETIVO: Verificar a variação sazonal de Aedes aegypti e Aedes albopictus e testar a associação da abundância das espécies com fatores abióticos.

MÉTODOS: De novembro de 2002 a outubro de 2003 foram realizadas coletas mensais de imaturos de culicídeos em pneus-armadilha expostos por 15 dias em área urbana de Potim, Vale do Paraíba, SP, Brasil. Os imaturos foram criados em laboratório por 29 dias e identificados segundo espécie. A associação com temperatura, pluviosidade e umidade relativa do ar foram testadas utilizando-se correlações de Spearman $\left(\mathrm{r}_{\mathrm{s}}\right)$. As estatísticas descritivas foram apresentadas pela média e erro-padrão (EP) e nos testes foi utilizado $\alpha=0,05$.

RESULTADOS: Do total de 20.727 imaturos coletados, 95,3\% eram Ae. aegypti e 4,7\% Ae. albopictus. A espécie Ae. aegypti esteve presente em todas as estações/ meses do ano e Ae. albopictus somente de novembro a julho. As associações das espécies coletadas com fatores abióticos foram significantes em relação à temperatura máxima para Ae. aegypti $(\mathrm{p}=0,04)$ e Ae. albopictus $(\mathrm{p}=0,01)$, e pluviosidade $(\mathrm{p}=0,02)$ para esta última espécie.

CONCLUSÕES: Ambas espécies apresentaram variação sazonal. Porém, Ae. aegypti esteve presente durante todo ano, com maiores quedas de densidade entre abril e maio e entre junho e julho. Ae. albopictus menos abundante, foi encontrado apenas de novembro a julho, com o pico em abril. A maior abundância de Ae. aegypti em relação à Ae. albopictus em área urbana mostrou maior capacidade de Ae. aegypti em colonizar pneus. A existência de tal criadouro no ambiente pode ser importante na manutenção e abundância de Ae. aegypti.

DESCRITO RES: Aedes aegypti. Aedes albopictus. Densidade demográfica. Distribuição temporal. Fatores abióticos.

Superintendência de Controle de Endemias. Taubaté, SP, Brasil

"Departamento de Biologia. Universidade de Taubaté. Taubaté, SP, Brasil

III Departamento de Epidemiologia. Faculdade de Saúde Pública, U niversidade de São Paulo. São Paulo, SP, Brasil

Correspondência | Correspondence: Lígia Leandro Nunes Serpa

Praça Coronel Vitoriano, 23 Jd. Santa Clara

12020-020 Taubaté, SP, Brasil

E-mail: ligiaserp@sucen.sp.gov.br

Recebido: 18/8/2005 Revisado: 9/6/2006 Aprovado: 12/7/2006 
correlations $\left(\mathrm{r}_{\mathrm{s}}\right)$. The descriptive statistics were presented by the average and the standard error $(\alpha=0.05)$.

RESULTS: From a total of 20,727 immatures collected, 95.3\% were Ae. aegypti and 4.7\%, Ae. albopictus. Ae. aegypti was found throughout the whole year while Ae. albopictus was found from November to July. The association between these species and abiotic factors was significant for Ae. aegypti $(\mathrm{p}=0.04)$ as for maximum temperature and for Ae. albopictus as for maximum temperature $(\mathrm{p}=0.01)$ and rainfall $(\mathrm{p}=0.02)$.

CONCLUSIONS: Both species presented seasonal variation. However, Ae. aegypti was found throughout the whole year showing a decrease between April and May and June and July. Ae. albopictus, less abundant, was found only between November, 2002 and July, 2003 with a peak in April, 2003. The higher abundance of Ae. aegypti compared to Ae. albopictus in an urban area showed Ae. aegypti has greater capacity of colonizing tires. Such a breeding site in the environment may be important to the maintenance and abundance of Ae. aegypti.

KEYW O RD S: Aedes aegypti. Aedes albopictus. Population density. Temporal distribution. Abiotic factors.

\section{INTRO DUÇÃO}

Culicídeos da ordem Diptera são mosquitos relevantes na epidemiologia de doenças transmitidas por vetores, visto seu papel na transmissão de praticamente metade dos Flavivirus. ${ }^{4}$ Dentre as enfermidades veiculadas pelas espécies Aedes (Stegomyia) aegypti (Linnaeus 1762) e Aedes (Stegomyia) albopictus (Skuse 1894), destacam-se dengue e febre amarela. A dengue é endêmica em todos os continentes, exceto na Europa, e possui maior importância epidemiológica. Nas Américas, surtos anuais vêm ocorrendo desde a década de 70 . Por outro lado, a febre amarela, que tem se apresentado na sua forma silvestre com casos esporádicos, encontra nas cidades das Américas áreas de grande risco do ressurgimento de epidemias urbanas devido à reinfestação por Ae. aegypti. ${ }^{5}$

Tais vetores tiveram sua introdução no Brasil em momentos históricos diferentes, Ae. aegypti no período da colonização e Ae. albopictus há pouco mais de duas décadas. A infestação destes Culicidae no Estado de São Paulo ocorreu nos anos 1980 enquanto que a sobreposição dessas espécies passou a ser registrada somente no final dessa década. ${ }^{7}$ A região do Vale do Paraíba, sul do Estado de São Paulo, tornou-se infestada primeiramente pela espécie Ae. albopictus, em 1986.* No ano de 2000, foi assinalada a infestação por Ae. aegypti no município de Potim, SP, onde casos de dengue passaram a ser registrados a partir de 2002. A dispersão de Ae. aegypti atingiu mais dois municípios da região nos anos subseqüen- tes, incluindo Aparecida, município contíguo a Potim, que alberga o maior santuário do Brasil e onde também se observou sobreposição com Ae. albopictus.* A cidade Aparecida ampliou sua infra-estrutura para integrar o roteiro do turismo católico internacional e recebe cerca de 7,5 milhões de peregrinos anualmente. Tal fato pode vir a incrementar o risco de transmissão da dengue, tornando vulnerável não apenas a população residente na região, assim como de outras áreas. Em conseqüência do processo de urbanização acelerado e aumento populacional dos centros urbanos, a presença de tais mosquitos é favorecida pela oferta de criadouros potenciais, sejam eles naturais ou artificiais.

Estudar a abundância e a freqüência destas espécies é importante para o entendimento dos mecanismos básicos de sua distribuição espacial com implicações práticas no controle. ${ }^{2}$ Assim, o presente estudo teve o objetivo de verificar a variação sazonal de Ae. aegypti e Ae. albopictus, além de investigar a influência de fatores abióticos como a temperatura, umidade relativa e pluviosidade sobre a abundância dessas espécies.

\section{MÉTODOS}

A área de estudo compreendeu o município de Potim, Vale do Paraíba, São Paulo, Brasil (2250’34”S e $45^{\circ} 15^{\prime} 05^{\prime}$ 'W), situado à margem esquerda da Rodovia Presidente Dutra, no eixo São Paulo-Rio de Janeiro, e também à margem esquerda do Rio Paraíba do Sul. Localiza-se a $535 \mathrm{~m}$ de altitude, ocupa $44,6 \mathrm{~km}^{2}$, com 12.955 habitantes na área urbana. 
Tabela - Número de pupas de Aedes aegypti e Aedes albopictus e informação relativa aos fatores abióticos do município. Potim, SP, 2002-2003.

\begin{tabular}{|c|c|c|c|c|c|c|c|}
\hline \multirow[t]{2}{*}{ M ês/ano } & \multirow{2}{*}{$\begin{array}{c}\text { Aedes aegypti } \\
\text { Número de pupas }\end{array}$} & \multirow{2}{*}{$\begin{array}{l}\text { Aedes albopictus } \\
\text { Número de pupas }\end{array}$} & \multirow{2}{*}{$\begin{array}{l}\text { Pluviosidade } \\
\text { mm }\end{array}$} & \multicolumn{2}{|c|}{ Temperatura ${ }^{\circ} \mathrm{C}$} & \multicolumn{2}{|c|}{ U midade } \\
\hline & & & & Média & Máxima & Mínima & Relativa \% \\
\hline $\begin{array}{l}\mathrm{N} \text { ov/02 } \\
\mathrm{D} \text { ez/02 } \\
\text { Jan/03 } \\
\mathrm{Fev} / 03 \\
\mathrm{Mar} / 03 \\
\mathrm{Abr} / 03 \\
\mathrm{Mai} / 03 \\
\mathrm{Jun} / 03 \\
\text { Jul/03 } \\
\text { Ago/03 } \\
\mathrm{Set} / 03 \\
\text { Out/03 }\end{array}$ & $\begin{array}{c}831 \\
590 \\
1.589 \\
1.959 \\
1.851 \\
3.048 \\
1.783 \\
2.208 \\
1.085 \\
1.367 \\
1.797 \\
1.641\end{array}$ & $\begin{array}{c}1 \\
49 \\
170 \\
35 \\
182 \\
484 \\
53 \\
3 \\
1 \\
0 \\
0 \\
0\end{array}$ & $\begin{array}{c}68,7 \\
86,7 \\
243,6 \\
43,3 \\
121,9 \\
25,1 \\
24,0 \\
3,8 \\
8,5 \\
2,8 \\
3,7 \\
27,6\end{array}$ & $\begin{array}{l}25 \\
26 \\
25 \\
30 \\
23 \\
23 \\
20 \\
21 \\
18 \\
20 \\
20 \\
23\end{array}$ & $\begin{array}{l}29 \\
34 \\
30 \\
36 \\
30 \\
31 \\
28 \\
29 \\
26 \\
24 \\
25 \\
27\end{array}$ & $\begin{array}{l}18 \\
20 \\
19 \\
20 \\
18 \\
17 \\
13 \\
14 \\
11 \\
11 \\
12 \\
15\end{array}$ & $\begin{array}{l}72 \\
72 \\
80 \\
76 \\
78 \\
71 \\
71 \\
72 \\
73 \\
70 \\
68 \\
68\end{array}$ \\
\hline
\end{tabular}

O estudo foi realizado no período de novembro de 2002 a outubro de 2003. Foram selecionadas 15 quadras, conforme capacidade operacional disponível, após estudo piloto. A seleção de quadras e imóveis ocorreu em dois estágios. No primeiro, as quadras a serem pesquisadas foram identificadas utilizando-se amostragem sistemática.* No segundo estágio, os imóveis a serem trabalhados foram localizados segundo a presença ou ausência de vegetação, locais de abrigo para os mosquitos. Foram feitas coletas mensais de formas imaturas em 15 pneus-armadilha, seccionados radialmente, expostos por 15 dias e instalados nas 15 quadras sorteadas da área urbana do município. Realizou-se uma coleta a cada 30 dias, totalizando 12 coletas ao final do trabalho.

As armadilhas foram instaladas no jardim, ou próximo a ele, a 1,5 m de altura, em local exposto ao sol no período da manhã e sombreado no período da tarde. $\mathrm{O}$ pneu foi fixado na face interna do muro frontal da residência e em todas as coletas eles eram escovados, lavados e flambados. Em cada pneu era introduzido 1,5 1 de água de fonte natural previamente submetida a uma rede de tamisação, malha $0,105 \mathrm{~mm}$, garantindo assim a retenção de ovos e larvas porventura presentes.

Decorridos sete dias de exposição, as armadilhas eram visitadas para verificação da presença e coleta apenas de larvas de terceiro e quarto estádio e de pupas. Os demais estádios, quando presentes, eram mantidos nas armadilhas para coleta no $15^{\circ}$ dia, período final da exposição. Desta forma pretendeu-se evitar tanto a perda de material pela emergência de adultos, como a manutenção de criadouro no ambiente. Procurou-se manter o volume de água das armadilhas constante, com reposição até 1,51.

No $15^{\circ}$ dia após a instalação, o volume total do pneu era recolhido, levado ao laboratório e passado em rede de tamisação. Os ovos e larvas retidos eram transferidos para cubas de vidro contendo um litro de água destilada e as larvas receberiam como alimento, ra- ção para peixes tropicais. As pupas formadas eram coletadas e fixadas em álcool a $70 \%$ e identificadas segundo espécie.*

Para cada evento de coleta, o período de criação em laboratório foi de 29 dias e fotoperíodo de 14L:10E, em temperatura ambiente. Ao final deste, as larvas existentes eram desprezadas.

Os dados de fatores abióticos investigados (temperatura, pluviosidade e umidade relativa do ar) foram fornecidos pelo Centro de Previsão de Tempo e Estudos Climáticos (CPTEC), do Instituto Nacional de Pesquisas Espaciais (INPE), Estação Meteorológica - Cachoeira Paulista, São Paulo.

Para testar a associação com temperatura, pluviosidade e umidade relativa do ar, foram utilizadas correlações não paramétricas de Spearman $\left(\mathrm{r}_{\mathrm{s}}\right)$. As estatísticas descritivas foram apresentadas pela média e erro-padrão (EP) e nos testes foi utilizado $\alpha=0,05$.

\section{RESU LTADOS}

Os dados relativos aos fatores abióticos e os números de espécimes de Aedes coletados a cada mês estão apresentados na Tabela.

Foram coletados 20.727 imaturos de Culicídeos, dos quais 19.749 (95,3\%) espécimes de Ae. aegypti e 978 (4,7\%) de Ae. albopictus. O número médio de $A e$. aegypti $(1561 \pm 192)$ foi maior $(\mathrm{t}=7,58 ; \mathrm{p}=0,0001)$ do que de Ae. albopictus (76 \pm 38$)$.

A Figura mostra a distribuição mensal de Ae. aegypti e Ae. albopictus. A presença de Ae. aegypti foi registrada do primeiro ao último mês de observação com pico no mês de abril, e apresentaram as maiores quedas entre abril e maio e entre junho e julho. Os menores números de mosquitos foram encontrados nos meses de julho, novembro e dezembro. Ae. albopictus, menos abundante, foi encontrado apenas de no- 


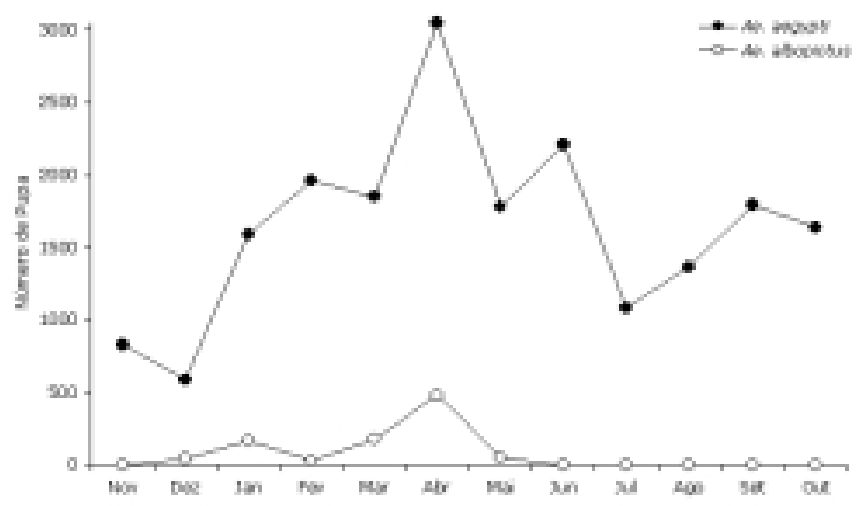

Figura - Distribuição mensal do número de pupas de Aedes aegypti e Aedes albopictus. Novembro de 2002 a outubro de 2003, município de Potim, SP.

vembro de 2002 a julho de 2003 com o pico em abril (Tabela, Figura).

As associações das espécies coletadas com fatores abióticos foram significantes para alguns fatores. Registrou-se associação significativa com temperatura máxima para Ae. aegypti $(\mathrm{p}=0,04)$ e Ae. albopictus ( $\mathrm{p}=0,01)$ e pluviosidade somente para Ae. albopictus $(\mathrm{p}=0,02)$. Esta espécie também registrou valores de associação mais altos com pluviosidade e temperatura máxima, $\mathrm{r}_{\mathrm{s}}=0,65$ e $\mathrm{r}_{\mathrm{s}}=0,69$, respectivamente, do que Ae. aegypti $\left(\mathrm{r}_{\mathrm{s}}=0,60\right)$. Não foi detectada associação de variação da umidade relativa com a abundância das espécies.

\section{DISCUSSÃO}

A abundância das populações de mosquitos pode sofrer influência de fatores bióticos e abióticos, potencialmente envolvidos com flutuações das populações. ${ }^{3}$ Embora a maioria dos mecanismos específicos envolvidos na abundância e variação sazonal dessas espécies não tenha feito parte da presente investigação, alguns aspectos serão discutidos.

Ae. aegypti apresentou-se como a espécie mais freqüente e abundante durante todas as estações do ano e na totalidade dos pneus pesquisados. Além disso, apresentou-se, em alguns meses, como espécie exclusiva nos pneus pesquisados. Os meses de julho, novembro e dezembro foram os de menor abundância para essa espécie. Embora Ae. aegypti tenha ocorrido em todas as estações do ano, observou-se redução nos meses de seca, conforme verificado anteriormente. ${ }^{11,12}$

Ae. albopictus apresentou-se em número reduzido e a maior quantidade de espécimes foi coletada entre janeiro e abril. Sua presença pouco expressiva em área urbana, habitat de escolha de Ae. aegypti, corro- bora outros estudos. ${ }^{15}$ Ae. albopictus foi mais sensível aos fatores ambientais, apresentando correlação significativa para pluviosidade e temperatura máxima.

Para ambas espécies foi registrado pico no mês de abril, o qual não correspondeu ao de maior pluviosidade. Similarmente, Gomes et $\mathrm{al}^{8}$ (1992) observaram pico nos meses de março e abril no estudo de microhabitats de Ae. albopictus em diferentes tipos de ambientes da mesma região.

Forattini \& Brito $^{6}$ (2003) mencionaram que a existência de reservatórios domésticos pode fornecer condições propícias à manutenção de populações de Ae. aegypti, mesmo em períodos de baixas precipitações. Esses autores relataram que caixas d'água foram os reservatórios preferenciais para o encontro de formas imaturas dessas duas espécies, nesta mesma área. Por esta razão, a presença de Ae. aegypti neste período deve ser analisada com cautela, uma vez que em áreas onde caixas d'água representam importantes criadouros de Ae. aegypti é necessário o desenvolvimento de estratégias mais eficientes de controle. Além disso, Kuno ${ }^{11}$ (1995) relatou a ocorrência de epidemias de dengue em épocas secas do ano, reforçando os achados.

Juliano et $\mathrm{al}^{9}$ (2002) mencionaram que períodos de seca causam maior mortalidade de ovos de Ae. albopictus quando comparados a Ae. aegypti. Portanto, a baixa frequiência de Ae. albopictus possivelmente seja justificada, em parte, pela menor resistência de seus ovos à dessecação.

Estudo $^{12}$ realizado na Tailândia, utilizando ovitrampa, revelou ausência de ovos de Ae. albopictus e presença de $A e$. aegypti, no final da estação seca. No referido estudo foi observado incremento considerável daquela espécie na estação chuvosa, verificando-se variação sazonal na composição destas espécies em área rural, bem como ocorrência irregular em área urbana. No presente estudo observou-se aumento gradativo de Ae. albopictus nos pneus durante o verão, exceto no mês de fevereiro. Neste mês, o índice pluviométrico registrado não foi o menor do período de estudo, mas reduzido quando comparado aos meses anterior e posterior.

Embora sem significância estatística, Ae. albopictus apresentou correlação de Spearman $\left(\mathrm{r}_{\mathrm{s}}=0,54\right)$ maior do que Ae. aegypti, para umidade relativa do ar. Segundo Mogi et al $^{13}$ (1996), a distribuição e abundância de espécies Ae. (Stegomyia) pode ser influenciada pela resistência dos adultos à dessecação. 
Sob o aspecto da coexistência dessas espécies em criadouros, estudos em diferentes regiões geográficas têm sugerido diferença na capacidade competitiva. Moore \& Fisher $^{14}$ (1969) observaram, por meio de experimento de competição em laboratório, o melhor desempenho de Ae. aegypti mesmo em baixos níveis de densidade larval. Esses autores sugeriram que a presença de inibidores químicos de crescimento pode ser importante na ecologia de ocupação de habitats onde tais metabólitos facilmente se acumulam, como por exemplo, em pneus descartados com larvas de mosquito. Entretanto, recentemente, foi confirmada a superioridade competitiva de larvas de Ae. albopictus em relação à Ae. aegypti em condições de campo, sugerindo que o impacto desse fenômeno biológico pode variar sazonalmente, associado à densidade de imaturos no criadouro. ${ }^{10}$ No presente estudo, o número de espécimes coletados na estação chuvosa foi praticamente o mesmo da estação seca.

\section{REFERÊNCIAS}

1. Barrera R. Competition and resistance to starvation in larvae of container-inhabiting Aedes mosquitoes. Ecol Entomol. 1996;21:117-27.

2. Braks MAH, Honório NA, Lourenço de Oliveira R, Juliano SA, Lounibos LP. Convergent habitat segregation of Aedes aegypti and Aedes albopictus (Diptera: Culicidae) in Southeastern Brazil and Florida. J Med Entomol. 2003;40:785-94.

3. Chapman HC, Barr AR, Laird M, Weidhaas DE. Biological control of mosquitos. J Am Mosq Control Assoc. 1985;6:185-94.

4. Consoli RAGB, Lourenço de Oliveira R. Principais mosquitos de importância sanitária no Brasil. Rio de Janeiro: Fiocruz; 1994.

5. Forattini OP. Culicidologia médica: identificação, biologia, epidemiologia. São Paulo: EDUSP; 2002. v. 2.

6. Forattini $O P$, Brito $M$. Reservatórios domiciliares de água e controle do Aedes aegypti. Rev Saúde Pública. 2003;37:676-7.

7. Glasser CM, Gomes AC. Clima e sobreposição da distribuição de Aedes aegypti e Aedes albopictus na infestação do estado de São Paulo. Rev Saúde Pública. 2002;36:166-72.

8. Gomes AC, Forattini OP, Kakitani I, Marques GRAM, Marques CCA, Marucci $D$, et al. Microhabitats de Aedes albopictus (Skuse) na região do Vale do Paraíba, estado de São Paulo, Brasil. Rev Saúde Pública. 1992;26:108-18.
Em condições de alta densidade larval Barrera ${ }^{1}$ (1996) verificou melhor desempenho de Ae. albopictus quando na presença de Ae.aegypti, sugerindo maior resistência da primeira espécie à superpopulação. No estudo de segregação de habitat,

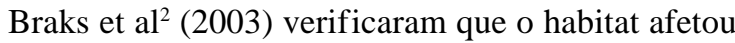
a abundância de Ae. aegypti e de Ae. albopictus, com predominância de Ae. aegypti em áreas altamente urbanizadas e Ae. albopictus em áreas rurais. Tais autores verificaram abundância similar de ambas as espécies na maior parte das áreas suburbanas estudadas.

A maior abundância de Ae. aegypti em relação à $A e$. albopictus em área urbana, inicialmente infestada por esta última espécie, mostrou maior capacidade de $A e$. aegypti em colonizar pneus. A existência de tal criadouro no ambiente pode ser importante na manutenção e abundância de Ae. aegypti.

9. Juliano SI, O 'M eara GI, Morrill JI, Cutwa MI. Desiccation and thermal tolerance of eggs and the coexistence of competing mosquitoes. Oecologia. 2002;130:458-69.

10. Juliano SI, Lounibos LP, O 'M eara GF. A field test for competitive effects of Aedes albopictus on A. aegypti in South Florida: differences between sites of coexistence and exclusion? Oecologia. 2004;139:583-93.

11. Kuno $G$. Review of the factors modulating dengue transmission. Epidemiol Rev. 1995;17:321-35.

12. Mogi M, Khamboonruang C, Choochote W, Suwanpanit $P$. O vitrap surveys of dengue vector mosquitoes in Chiang Mai, northern Thailandia: seasonal shifts in relative abundance of Aedes albopictus and Aedes aegypti. Med Vet Entomol. 1988;2:319-24

13. Mogi M, Miyagi I, Abadi K, Syafruddin. Inter- and intraspecific variation in resistaence to desiccation by adult Aedes (Stegomyia) spp. (Diptera:Culicidae) from Indonesia. J Med Entomol. 1996;33:53-7.

14. Moore CG, Fisher BR. Competition in mosquitoes: density and species ratio effects on growth, mortality, fecundity, and production of growth retardant. Ann Entomol Soc Am. 1969;62:1325-31.

15. Passos RA, Marques GRAM, Voltolini JC, Condino MLF. Dominância de Aedes aegypti sobre Aedes albopictus no litoral sudeste do Brasil. Rev Saúde Pública. 2003;37:729-34.

Trabalho realizado no Laboratório de Culicídeos, Superintendência de Controle de Endemias (Taubaté), e no Núcleo de Pesquisa Taxonômica e Sistemática em Entomologia Médica (NUPTEM) da Universidade de São Paulo.

Financiado pela Fundação de Amparo à Pesquisa do Estado de São Paulo (Fapesp - Processo n: Temático 99/10517-1). 